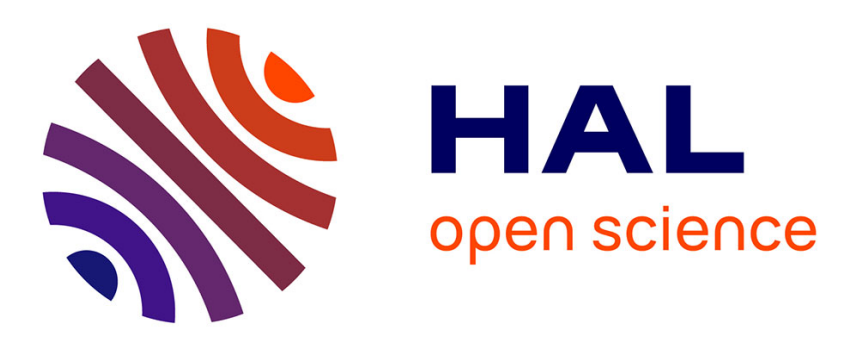

\title{
Theoretical basis of regularized integral equations for elastostatic crack problems
}

\author{
Anh Le Van, Jean Royer
}

\section{To cite this version:}

Anh Le Van, Jean Royer. Theoretical basis of regularized integral equations for elastostatic crack problems. International Journal of Fracture, 1990, 44, pp.155-166. 10.1007/BF00035513 . hal01007380

\section{HAL Id: hal-01007380 \\ https://hal.science/hal-01007380}

Submitted on 9 Oct 2016

HAL is a multi-disciplinary open access archive for the deposit and dissemination of scientific research documents, whether they are published or not. The documents may come from teaching and research institutions in France or abroad, or from public or private research centers.
L'archive ouverte pluridisciplinaire HAL, est destinée au dépôt et à la diffusion de documents scientifiques de niveau recherche, publiés ou non, émanant des établissements d'enseignement et de recherche français ou étrangers, des laboratoires publics ou privés. 


\title{
Theoretical basis of regularized integral equations for elastostatic crack problems
}

\author{
A. LEVAN and J. ROYER \\ Laboratoire de Mécanique des Structures, Ecole Nationale Supérieure de Mécanique, Nantes 44072 Cedex, \\ France
}

\begin{abstract}
Integral equations derived by means of the potential theory for statical crack problems are singular in the sense of the principal value. In the present paper, these integrals are transformed into weakly singular ones and the so-called regularized integral equation is thus obtained. The conditions which permit the transformation are discussed and the weak singularity is proved. The kernel of the regularized equation is written in terms of the density, equal to the displacement discontinuity on the crack surface, in such a way that no extension of this density is involved. The results obtained hold for either embedded or surface crack problems.
\end{abstract}

\section{Introduction}

Integral equations proposed for crack problems, or more generally for various problems within the context of the potential theory, have the specific feature that they are singular in the sense of the principal value. This singularity entailing some difficulties especially for numerical investigations necessitates the study of the regularization procedure with the view of obtaining equations containing integrals with weakly singular kernels, commonly referred to as regularized integral equations.

In elastostatics related to non-cracked body problems, the Somigliana formulation leads to a singularity which is removed analytically in [1], where the boundary surface is considered as made up of planar elements and the collocation point taken at the center of each element. Later on, the regularization is carried out under more general hypotheses in [2] in the case of finite bodies. The results are generalized to the case of infinite media in [3]. In elastodynamics, the solution is less obvious since there no longer exists a solution of the rigid displacement type which allowed the extraction of the singular part. The elastodynamic problems were successfully solved in [3-6].

As regards crack problems, the regularized integral equations have recently been obtained in [7] for two-dimensional curve cracks, in [3] and [8] for three-dimensional cracks.

Comprised in this paper is a further investigation within the scope of elastostatics, of the study in [3]. After a brief review of the singular integral for static crack problems, it is transformed, on the basis of the method described in [3], into a sum of weakly singular or ordinary improper integrals. The hypotheses which enable this transformation are explicitly stated and the nature of some weakly singular integrals is proved.

As in [9], another expression for the regularized integral is proposed, whose kernel now involves the density function itself, and no longer its extension. In the case of a twice continuously differentiable crack surface, the principal curvature radii are shown to appear, either coupled or uncoupled. 
It should be noticed that in this study as well as in other works, the regularization techniques do not actually yield regular integrals, but only ordinary improper ones.

In the following study, Latin indices take the value 1,2 or 3, Greek indices the value 1 or 2 , and implicit sums are implied on any repeated index.

\section{Theoretical background of singular integral equations $[9,10]$}

Consider a three-dimensional cracked body filled with a linear elastic material. The crack is represented by a surface $S$ in the space $E_{3}$ such that $S \in C^{1, \alpha}, 0<\alpha \leqslant 1$. In this study, the crack edge $\partial S$ is considered as excluded from $S$, i.e., $S \backslash \partial S=S$. The displacement field is defined in $E_{3} \backslash S$ by:

$$
\forall x \in E_{3} \backslash S, \mathbf{u}(x)=\int_{S} \mathbf{T}^{T}\left(y-x, \mathbf{n}_{y}\right) \varphi(y) \mathrm{d}_{y} S,
$$

where $\varphi: S \rightarrow \mathbb{R}^{3}$ referred to as the density is a function defined on $S$, satisfying the assumption:

$$
\varphi \in C^{1, \beta}(S), \quad 0<\beta \leqslant 1 .
$$

T denotes the Kupradze tensor [11]:

$$
\begin{aligned}
\mathbf{T}\left(y-x, \mathbf{n}_{y}\right)= & \frac{1}{8 \pi(\lambda+2 \mu) r^{2}} \\
& \times\left\{2 \mu\left[\mathbf{n}_{y} \otimes \mathbf{e}_{r}-\mathbf{e}_{r} \otimes \mathbf{n}_{y}-\left(\mathbf{e}_{r} \mathbf{n}_{y}\right) \mathbf{I}-6(\lambda+\mu)\left(\mathbf{n}_{y} \mathbf{e}_{r}\right) \mathbf{e}_{r} \otimes \mathbf{e}_{r}\right\} .\right.
\end{aligned}
$$

$r=|y-x|, \mathbf{e}_{r}=(y-x) / r, \mathbf{I}$ is the unit tensor.

The displacement field $\mathbf{u}$ defined in (1) is a possible displacement field as proved in [11], and it is continuous everywhere in $E_{3}$ except on $S$. Under the previous hypotheses on $S$ and $\varphi$, the displacement discontinuity through the crack surface is given by:

$$
\forall y_{0} \in S, \quad\left[\mathbf{u}\left(y_{0}\right)\right] \equiv \mathbf{u}\left(y_{0}^{+}\right)-\mathbf{u}\left(y_{0}^{-}\right)=\boldsymbol{\varphi}\left(y_{0}\right) .
$$

At any point $x \in E_{3} \backslash S$, the resulting stress vector related to the normal $\mathbf{n}_{x}$ is given by:

$$
\mathbf{t}\left(x, \mathbf{n}_{x}\right)=\mathscr{T}\left(\partial x, \mathbf{n}_{x}\right) \mathbf{u}(x)=\mathscr{T}\left(\partial x, \mathbf{n}_{x}\right) \int_{S} \mathbf{T}^{T}\left(y-x, \mathbf{n}_{y}\right) \varphi(y) \mathrm{d}_{y} S,
$$

where $\mathscr{T}\left(\partial x, \mathbf{n}_{x}\right)$ denotes the stress operator:

$$
\begin{aligned}
\mathscr{T}\left(\partial x, \mathbf{n}_{x}\right) & =2 \mu \mathrm{d} / \mathrm{d} n_{x}+\mu \mathbf{n}_{x} \wedge \mathbf{r o t} t_{x}+\lambda \mathbf{n}_{x} \operatorname{div}_{x}, \\
\mathscr{T}_{i k}\left(\partial x, \mathbf{n}_{x}\right) & =c_{i j k l} n_{j}(x) \partial / \partial x_{l} \quad\left(c_{i j k l}=\lambda \delta_{i j} \delta_{k l}+\mu\left(\delta_{i k} \delta_{j l}+\delta_{i l} \delta_{k j}\right)\right),
\end{aligned}
$$

the stress operator $\mathscr{T}$ is related to the Kupradze tensor $\mathbf{T}$ by:

$$
\mathbf{T}\left(z-x, \mathbf{n}_{y}\right)=\mathscr{T}\left(\partial z, \mathbf{n}_{y}\right) \mathbf{E}(z-x)
$$


where $\mathbf{E}$ is the Kelvin tensor:

$$
\begin{aligned}
& \mathbf{E}(z-x)=\frac{1}{8 \pi(\lambda+2 \mu) r}\left[(\lambda+3 \mu) \mathbf{I}+(\lambda+\mu) \mathbf{e}_{r} \otimes \mathbf{e}_{r}\right], \\
& r=|z-x|, \quad \mathbf{e}_{r}=(z-x) / r .
\end{aligned}
$$

Relation (2) combined with (3) then gives the stress vector:

$$
\mathbf{t}\left(x, \mathbf{n}_{x}\right)=\left.\mathscr{T}\left(\partial x, \mathbf{n}_{x}\right) \int_{S}\left[\mathscr{T}\left(\partial z, \mathbf{n}_{y}\right) \mathbf{E}(z-x)\right]^{T}\right|_{z=y} \boldsymbol{\varphi}(y) \mathrm{d}_{y} S
$$

and its $l$-th component:

$$
t_{l}\left(x, \mathbf{n}_{x}\right)=\left.\mathscr{T}_{l j}\left(\partial x, \mathbf{n}_{x}\right) \int_{S}\left[\mathscr{T}_{i k}\left(\partial z, \mathbf{n}_{y}\right) E_{k j}(z-x)\right]\right|_{z=y} \varphi_{i}(y) \mathrm{d}_{y} S
$$

on account of: $\left(\partial E_{i j} / \partial x_{k}\right)(z-x)=-\left(\partial E_{i j} / \partial z_{k}\right)(z-x)$, we have:

$$
t_{l}\left(x, \mathbf{n}_{x}\right)=-\left.c_{l p j q} c_{i s k t} n_{p}(x) \int_{S} \frac{\partial}{\partial z_{\mathrm{q}}} \frac{\partial E_{k j}(z-x)}{\partial z_{t}}\right|_{z=y} n_{s}(y) \varphi_{i}(y) \mathrm{d}_{y} S .
$$

One can transform the kernel of the surface integral in relation (4):

$$
\left.n_{s}\left(E_{k j, t}\right)_{, q}\right|_{z=y} \varphi_{i}(y)=\left.\mathscr{D}_{s q}\left(E_{k j, t} \tilde{\varphi}_{i}\right)\right|_{z=y}-\left.\left(E_{k j, t} \mathscr{D}_{s q} \tilde{\varphi}_{i}\right)\right|_{z=y}+\left.n_{q}(y) E_{k j, s t}\right|_{z=y} \varphi_{i},
$$

where we have denoted for brevity:

$$
\cdot_{, i} \equiv \partial . / \partial z_{i} \quad \text { and }\left.\left.\quad \mathscr{D}_{j k} \tilde{\varphi}_{i} \equiv \mathscr{D}_{j k}\left(\partial z, n_{y}\right) \tilde{\varphi}_{i}(z)\right|_{z=y} \equiv n_{j}(y) \frac{\partial \tilde{\varphi}_{i}(z)}{\partial z_{k}}\right|_{z=y}-\left.n_{k}(y) \frac{\partial \tilde{\varphi}_{i}(z)}{\partial z_{j}}\right|_{z=y} .
$$

$\tilde{\varphi}_{i}$ is an extension of $\varphi_{i}$ to an open set including $S, \mathscr{D}_{j k}$ is the tangent operator (see the Appendix). Stokes' theorem applied to the first term of relation (5), together with the equilibrium equation, $\forall x \in S, c_{i s k t} E_{k j, s t}=0$, then yield a new expression of the stress vector:

$$
t_{l}\left(x, \mathbf{n}_{x}\right)=\left.c_{l p j q} c_{i s k t} n_{p}(x) \int_{S}\left(E_{k j, t} \mathscr{D}_{s q} \tilde{\varphi}_{i}\right)\right|_{z=y} d_{y} S+\left.c_{l p j q} c_{i s k t} n_{p}(x) \int_{S} \varepsilon_{m q s} \varphi_{i}(y) E_{k j, l}\right|_{z=y} \mathrm{~d} y_{m} .
$$

On the basis of relation (6), the stress vector at any point $y_{0} \in S$ is obtained by passing in (5) to the limit as $x \rightarrow y_{0}$. In $[9,10]$, it is shown that the stress vector has the same form as (5), provided the surface integral is taken in the sense of the principal value, denoted by the symbol $v p$ :

$$
\begin{aligned}
\forall y_{0} \in S, \quad t_{l}\left(y_{0}, \mathbf{n}_{y_{0}}\right)= & \left.c_{l p j q} c_{i s k t} n_{p}\left(y_{0}\right) v p \int_{S}\left(E_{k j, t} \mathscr{D}_{s q} \tilde{\varphi}_{i}\right)\right|_{z=y} \mathrm{~d}_{y} S \\
& +\left.c_{l p j q} c_{i s k l} n_{p}\left(y_{0}\right) \int_{\partial S} \varepsilon_{m q s} \varphi_{i}(y) E_{k j, t}\right|_{z=y} \mathrm{~d} y_{m} .
\end{aligned}
$$


If the stress is prescribed throughout the crack surface, relation (6) constitutes the singular integral equation for three-dimensional crack problems. The line integral in relation (7) is involved in problems of surface cracks or cracks with a common edge part. It requires no special study, since the edge $\partial S$ is not included in the crack surface and thus it remains in any case regular. On the other hand, the surface integral is singular, since the derivatives $E_{k j, t}$ behave like $r^{-2}, r=\left|y-y_{0}\right|$. In the following study, the surface integral in relation (7) will be transformed in order to obtain a regularized expression of the stress vector for any point of the crack.

\section{Regularized integral equation}

The principal value integral in relation (7) is rewritten as the sum of a weakly singular one (denoted by an asterisk) and another principal value one:

$$
\begin{aligned}
& \forall y_{0} \in S, \quad t_{l}\left(y_{0}, \mathbf{n}_{y_{0}}\right)=c_{l p j q} c_{i s k t} n_{p}\left(y_{0}\right)\left\{* \int_{S}\left[\left.\mathscr{D}_{j k}\left(\partial z, \mathbf{n}_{y}\right) \tilde{\varphi}_{i}(z)\right|_{z=y}-\left.\mathscr{D}_{j k}\left(\partial z, \mathbf{n}_{y_{0}}\right) \tilde{\varphi}_{i}(z)\right|_{z=y_{0}}\right]\right. \\
& \left.\quad \times\left.\frac{\partial E_{k j}\left(z-y_{0}\right)}{\partial z_{t}}\right|_{z=y} \mathrm{~d}_{y} S+\left.\left.v p \int_{S} \mathscr{D}_{j k}\left(\partial z, \mathbf{n}_{y_{0}}\right) \tilde{\varphi}_{i}(z)\right|_{z=y_{0}} \frac{\partial E_{k j}\left(z-y_{0}\right)}{\partial z_{t}}\right|_{z=y} \mathrm{~d}_{y} S\right\} \\
& \quad+\int_{\partial S} N_{o y}(\varphi)\left(y_{0}, y\right) \mathrm{d}_{y} l .
\end{aligned}
$$

For brevity, the kernel of the line integral in relation (7) has been denoted by $\operatorname{Noy}_{\partial S}(\varphi)\left(y_{0}, y\right)$. The nature of the first integral is a priori unknown, later it will be shown that this integral is actually weakly singular, and therefore the whole principal value singularity is transferred into the second integral. For the moment, in accordance with:

$$
\begin{aligned}
& \left.\frac{\partial E_{k j}\left(z-y_{0}\right)}{\partial z_{t}}\right|_{z=y}=-\frac{1+v}{8 \pi E(1-v) r^{2}}\left[(3-4 v) \delta_{k j} r_{, t}-\delta_{j t} r_{, k}-\delta_{k t} r_{, j}+3 r_{, k} r_{, j} r_{, t}\right], \\
& r=\left|y-y_{0}\right|, \quad r_{, i}=\left(y_{i}-y_{0 i}\right) / r,
\end{aligned}
$$

the first integral in relation (8) is:

$$
\begin{aligned}
& c_{l p j q} c_{i s k t} n_{p}\left(y_{0}\right) * \int_{S}=\frac{E n_{p}\left(y_{0}\right)}{16 \pi\left(1-v^{2}\right)} * \int_{S}\left\{4 v \delta_{l p} \Delta \mathscr{D}_{i k} \tilde{\varphi}_{i} r_{, k}+(1-2 v)\left[\Delta \mathscr{D}_{i p} \tilde{\varphi}_{i} r_{, l}+\Delta \mathscr{D}_{i l} \tilde{\varphi}_{i} r_{, p}\right.\right. \\
& \left.\left.+r_{, k}\left(\Delta \mathscr{D}_{p k} \tilde{\varphi}_{l}+\Delta \mathscr{D}_{l k} \tilde{\varphi}_{p}\right)\right]+3 r_{, i} r_{k}\left(\Delta \mathscr{D}_{p k} \tilde{\varphi}_{i} r_{, l}+\Delta \mathscr{D}_{l k} \tilde{\varphi}_{i} r_{, p}\right)\right\} \mathrm{d}_{y} S
\end{aligned}
$$

where:

$$
\left.\Delta \mathscr{D}_{s q} \tilde{\varphi}_{i} \equiv \mathscr{D}_{s q}\left(\partial z, \mathbf{n}_{y}\right) \tilde{\varphi}_{i}(z)\right|_{z=y}-\left.\mathscr{D}_{s q}\left(\partial z, \mathbf{n}_{y_{0}}\right) \tilde{\varphi}_{i}(z)\right|_{z=y_{0}} .
$$


Let us denote:

. $F: \Delta \ni u^{\prime} \rightarrow y^{\prime} \in S$, a (local or global) parametrization of $S\left(\Delta:\right.$ a domain in $\left.\mathbb{R}^{2}\right)$,

$. \phi=\varphi_{0} F: \Delta \ni u^{\prime} \rightarrow \phi\left(u^{\prime}\right) \in \mathbb{R}^{3}$, the composition of the parametrization $F$ and the vector density $\varphi$,

$. u, u_{0} \in \Delta$ : the antecedents by $\phi$ of $y, y_{0} \in S$, respectively.

Following the same reasoning as in [9], and making use of relation (A.4) in the Appendix, one can obtain a new expression for the difference $\Delta \mathscr{D}_{s q} \tilde{\varphi}_{i}$, where the extension function $\tilde{\varphi}_{i}$ is no longer involved:

$$
\begin{aligned}
\Delta \mathscr{D}_{s q} \tilde{\varphi}_{i}= & \varepsilon_{m q s}\left(\mathbf{e}_{s} \wedge \mathbf{e}_{q}\right)\left[\frac{\partial \phi_{i}}{\partial u_{\alpha}^{\prime}}(u) \frac{\partial \mathbf{F}}{\partial u_{\beta}^{\prime}}(u) \frac{1}{\left|\frac{\partial \mathbf{F}}{\partial u_{1}^{\prime}}(u) \wedge \frac{\partial \mathbf{F}}{\partial u_{2}^{\prime}}(u)\right|}\right. \\
& \left.-\frac{\partial \phi_{i}}{\partial u_{\alpha}^{\prime}}\left(u_{0}\right) \frac{\partial \mathbf{F}}{\partial u_{\beta}^{\prime}}\left(u_{0}\right) \frac{1}{\left|\frac{\partial \mathbf{F}}{\partial u_{1}^{\prime}}\left(u_{0}\right) \wedge \frac{\partial \mathbf{F}}{\partial u_{2}^{\prime}}\left(u_{0}\right)\right|}\right]
\end{aligned}
$$

Relation (9) proves to be useful since it will allow us to obtain the regularized stress vector without the presence of the extension function.

3.1. Why is the first integral in relation (8) an ordinary improper integral?

Now we come to prove that the first integral in relation (8) is actually ordinary improper, i.e. its kernel behaves like $r^{2-\gamma}, \gamma>0$, as the point $y$ tends to $y_{0}$. First, in accordance with the assumption $S \in C^{1, \alpha}, 0<\alpha \leqslant 1$, we have: $F \in C^{1, \alpha}$, then $\partial \mathbf{F} / \partial u_{\beta}^{\prime}$ and

$$
\frac{1}{\left|\frac{\partial \mathbf{F}}{\partial u_{1}^{\prime}}(u) \wedge \frac{\partial \mathbf{F}}{\partial u_{2}^{\prime}}(u)\right|} \in C^{0, \alpha} .
$$

On the other hand, by virtue of assumption $\varphi \in C^{1, \beta}(S), 0<\beta \leqslant 1$, we have: $\phi=$ $\varphi_{0} F \in C^{1, \beta}(S)$, then:

$\partial \phi / \partial u_{\alpha}^{\prime} \in C^{0, \beta}$.

One can deduce an upper bound for $\Delta \mathscr{D}_{s q} \tilde{\varphi}_{i}$ in relation (10)

$$
\begin{aligned}
\left|\Delta \mathscr{D}_{s q} \tilde{\varphi}_{i}\right| & \leqslant\left|u-u_{0}\right|^{\gamma} \quad(\gamma=\min (\alpha, \beta)) \\
& \leqslant\left|\eta-y_{0}\right|^{\gamma} \quad\left(\eta=\text { projection of } y \text { on the tangent plane at } y_{0} \text { of } S\right) \\
& \leqslant\left|y-y_{0}\right|^{\gamma}
\end{aligned}
$$


Inequality (11) shows that integral (9) is ordinary improper. Now the strong singularity is found to be transferred into the second integral in relation (8). This implies that the regularization task will consist of extracting the principal value in the last integral.

After performing all summations, the second integral in relation (8) becomes:

$$
\begin{aligned}
\left.\left.c_{l p j q} c_{i s k t} n_{p}\left(y_{0}\right) v p \int_{S} \mathscr{D}_{s q}\left(\partial z, \mathbf{n}_{y_{0}}\right) \tilde{\varphi}_{i}(z)\right|_{z=y_{0}} \frac{\partial E_{k j}\left(z-y_{0}\right)}{\partial z_{t}}\right|_{z=y} \mathrm{~d}_{y} S \\
=v p \int_{S}\left\{\left.4 v \delta_{l p} \mathscr{D}_{i k} \tilde{\varphi}_{i}\right|_{z=y_{0}} r_{, k}+(1-2 v)\left[\left.\mathscr{D}_{i p} \tilde{\varphi}_{i}\right|_{z=y_{0}} r_{, l}+\left.\mathscr{D}_{i l} \tilde{\varphi}_{i}\right|_{z=y_{0}} r_{, p}+r_{, k}\left(\left.\Delta \mathscr{D}_{p k} \tilde{\varphi}_{l}\right|_{z=y_{0}}\right.\right.\right. \\
\left.\left.\left.\quad+\left.\mathscr{D}_{l k} \tilde{\varphi}_{p}\right|_{z=y_{0}}\right)\right]+3 r_{, i} r_{, k}\left(\left.\mathscr{D}_{p k} \tilde{\varphi}_{i}\right|_{z=y_{0}} r_{, l}+\left.\mathscr{D}_{l k} \tilde{\varphi}_{i}\right|_{z=y_{0}} r_{, p}\right)\right\} \mathrm{d}_{y} S,
\end{aligned}
$$

where:

$$
\left.\left.\mathscr{D}_{i k} \tilde{\varphi}_{i}\right|_{z=y_{0}} \equiv \mathscr{D}_{i k}\left(\partial z, n_{y_{0}}\right) \tilde{\varphi}_{i}(z)\right|_{z=y_{0}} \cdot
$$

To remove the extension function $\tilde{\varphi}$ in the right hand side of relation (12), use will be made of:

$$
\left.\mathscr{D}_{i k}\left(\partial z, \mathbf{n}_{y_{0}}\right) \tilde{\varphi}_{i}(z) r_{, k}\right|_{z=y_{0}} \mathbf{n}_{y_{0}}=-\varepsilon_{3 \alpha \beta}\left(\frac{\partial \boldsymbol{\phi}}{\partial u_{\alpha}^{\prime}}\left(u_{0}\right), \frac{\partial \mathbf{F}}{\partial u_{\beta}^{\prime}}\left(u_{0}\right), \mathbf{e}_{r}\right) \mathbf{n}_{y_{0}} \frac{1}{\left|\frac{\partial \mathbf{F}}{\partial u_{1}^{\prime}}\left(u_{0}\right) \wedge \frac{\partial \mathbf{F}}{\partial u_{2}^{\prime}}\left(u_{0}\right)\right|}
$$

(all terms are calculated at $u_{0}$, the antecedent of point $y_{0}$ by $\phi$ ), which transforms the right-hand side of relation (12) into:

$$
\begin{aligned}
& c_{l p j q} c_{i s k t} n_{p}\left(y_{0}\right) v p \int_{S}=\frac{E}{16 \pi\left(1-v^{2}\right)} \varepsilon_{3 \alpha \beta}\left\{2\left(\mathbf{F}_{, \beta}\left(u_{0}\right), \phi_{, \alpha}\left(u_{0}\right), v p \int_{S} \frac{\mathbf{e}_{r}}{r^{2}} \mathrm{~d}_{y} S\right) \mathbf{n}_{y_{0}}\right. \\
& -(1-2 v)\left(\boldsymbol{\phi}_{, \alpha}\left(u_{0}\right), v p \int_{S} \frac{\mathbf{e}_{r}}{r^{2}} \mathrm{~d}_{y} S, \mathbf{n}_{y_{0}}\right) \mathbf{F}_{, \beta}\left(u_{0}\right) \\
& +3\left[v p \int_{S}\left(\mathbf{e}_{r} \boldsymbol{\phi}_{, \alpha}\left(u_{0}\right)\right)\left(\mathbf{F}_{, \beta}\left(u_{0}\right), \mathbf{n}_{y_{0}}, \mathbf{e}_{r}\right) \frac{\mathbf{e}_{r}}{r^{2}} \mathrm{~d}_{y} S\right. \\
& \left.\left.+\left(v p \int_{S}\left(\mathbf{e}_{r} \boldsymbol{\phi}_{, \alpha}\left(u_{0}\right)\right)\left(\mathbf{n}_{y_{0}} \mathbf{e}_{r}\right) \frac{\mathbf{e}_{r}}{r^{2}} \mathrm{~d}_{y} S\right) \wedge \mathbf{F}_{, \beta}\left(u_{0}\right)\right]\right\} \frac{1}{\left|\mathbf{F}_{, 1}\left(u_{0}\right) \wedge \mathbf{F}_{, 2}\left(u_{0}\right)\right|}
\end{aligned}
$$

where:

$$
\cdot_{, \alpha} \equiv \partial .\left|\partial u_{\alpha}^{\prime}, \quad r=\right| y-y_{0} \mid, \quad \mathbf{e}_{r}=\left(y-y_{0}\right) / r .
$$


Relation (14) gives a new expression of the second integral in relation (8) without the presence of the extension function $\tilde{\varphi}$. There enter at the same time integrals of the types:

$$
\begin{aligned}
& v p \mathbf{I}\left(y_{0}\right) \equiv v p \int_{S} \frac{\mathbf{e}_{r}}{r^{2}} \mathrm{~d}_{y} S, \\
& v p \mathbf{J}(\mathbf{a}, \mathbf{b})\left(y_{0}\right) \equiv v p \int_{S} \frac{1}{r^{2}}\left(\mathbf{a e}_{r}\right)\left(\mathbf{b e}_{r}\right) \mathbf{e}_{r} \mathrm{~d}_{y} S .
\end{aligned}
$$

$y_{0} \in S$, the vectors $\mathbf{a}, \mathbf{b}$ are constant with respect to the integration. Integrals (15) we first introduced in [12], their existence being due to the presence of vector $\mathbf{e}_{r}$ in (15) once or three times, so that the theorems in [11], pp. 87-89, [13], p. 2, hold. At this stage, it remains to regularize these singular integrals. For this, use will be made of the following lemmas related to the integrals:

$$
\begin{aligned}
& \mathbf{I}(x) \equiv \int_{S} \frac{\mathbf{e}_{r}}{r^{2}} \mathrm{~d}_{y} S, \\
& \mathbf{J}(\mathbf{a}, \mathbf{b})(x) \equiv \int_{S} \frac{1}{r^{2}}\left(\mathbf{a e}_{r}\right)\left(\mathbf{b e}_{r}\right) \mathbf{e}_{r} \mathrm{~d}_{y} S,
\end{aligned}
$$

with now:

$$
x \in E_{3} \backslash S, \quad r=|y-x|, \quad \mathbf{e}_{r}=(y-x) / r .
$$

The following lemma is proved in [12]:

Lemma 1: If $S \in C^{2}$, then integral (16a) can be transformed into:

$$
\mathbf{I}(x)=\int_{S}\left(\frac{\mathbf{n}_{y} \mathbf{e}_{r}}{r^{2}}-\frac{\kappa(y)}{r}\right) \mathbf{n}_{y} \mathrm{~d}_{y} S-\int_{\partial S} \frac{\boldsymbol{v}}{r} \mathrm{~d}_{y} l,
$$

where $\boldsymbol{v}=\tau \wedge \mathbf{n}_{y}, \tau$ is the vector tangent to the crack contour $\partial S, \kappa(y)$ the mean curvature, $=1 / R_{1}+1 / R_{2}, R_{1}, R_{2}$ are the principal curvature radii at $y$.

Let us prove the following lemma:

Lemma 2: If $S \in C^{2}$, then integral (16b) can be transformed into:

$$
\begin{aligned}
\mathbf{J}(\mathbf{a}, \mathbf{b})(x)= & \frac{1}{3}\left\{\int_{S}\left[\frac{3}{2}\left(\mathbf{e}_{r} \mathbf{S} \mathbf{e}_{r}\right) \mathbf{n}_{y}-\mathbf{n}_{y} \wedge\left(\mathbf{n}_{y} \wedge \mathbf{S n}_{y}\right)\right] \frac{\mathbf{e}_{r} \mathbf{n}_{y}}{r^{2}} \mathrm{~d}_{y} S\right. \\
& +\int_{S}\left[-\frac{1}{2}\left(\mathbf{e}_{r} \mathbf{S} \mathbf{e}_{r}\right) \mathbf{n}_{y}+\mathbf{n}_{y} \wedge\left(\mathbf{n}_{y} \wedge \mathbf{S n}_{y}\right)\right] \frac{\kappa(y)}{r} \mathrm{~d}_{y} S \\
& -\int_{S}\left[\sum_{\alpha=1}^{2} \frac{1}{R_{x}}\left(\mathbf{e}_{\alpha}^{y} \mathbf{S e}_{\alpha}^{y}\right) \mathbf{n}_{y}+\sum_{\alpha=1}^{2}\left(\mathbf{e}_{\alpha}^{y} \mathbf{S e}_{\alpha}^{y}\right) \frac{\mathbf{e}_{\alpha}^{y}}{R_{\alpha}}\right] \frac{1}{r} \mathrm{~d}_{y} S \\
& \left.+\int_{\partial S}\left[-\frac{1}{2}\left(\mathbf{e}_{r} \mathbf{S e}_{r}\right) \boldsymbol{v}+\mathbf{n}_{y} \wedge\left(\mathbf{n}_{y} \wedge \mathbf{S} \boldsymbol{v}\right)\right] \frac{1}{r} \mathrm{~d}_{y} l\right\},
\end{aligned}
$$


where $\mathbf{e}_{1}^{y}, \mathbf{e}_{2}^{y}$ denote the vector of the principal basis at $y$, related to the principal radii $R_{1}, R_{2}$; $\mathbf{S}$. designates the symmetrical tensor $\mathbf{a} \otimes \mathbf{b}+\mathbf{b} \otimes \mathbf{a}$.

Proof: Let us rewrite integral $\mathbf{J}$ as follows:

$$
\begin{aligned}
& \mathbf{J}(\mathbf{a}, \mathbf{b})(x)=\frac{1}{3}\left[\int_{S} \frac{\mathbf{b e}_{r}}{r^{2}} \mathrm{~d}_{y} S . \mathbf{a}+\int_{S} \frac{\mathbf{a e}_{r}}{r^{2}} \mathrm{~d}_{y} S . \mathbf{b}-\int_{S}\left(\frac{r_{, i} r_{, j}}{r}\right)_{, k} \mathrm{~d}_{y} S . a_{i} b_{j} \mathbf{e}_{k}\right], \\
& r_{, i}=\partial|z-x| /\left.\partial z_{i}\right|_{z=y}=\left(y_{i}-x_{i}\right) / r .
\end{aligned}
$$

As the first two integrals relate to integral $\mathbf{I}(x)$ already studied in Lemma 1 above, it remains for us to consider the last one. Using the operator $\mathscr{P}$ grad introduced in the Appendix, one has:

$$
\int_{S}\left(\frac{r_{, i} r_{, j}}{r}\right)_{, k} \mathrm{~d}_{y} S=\int_{S}\left[\mathscr{P} \operatorname{grad}_{k} \frac{r_{i,} r_{, j}}{r}+\frac{n_{k}}{r}\left(n_{i} r_{, j}+n_{j} r_{, i}-3 r_{, i} r_{, j}\left(\mathbf{e}_{r} \mathbf{n}_{y}\right)\right)\right] \mathrm{d}_{y} S .
$$

Now, denoting by $\tilde{n}: z \rightarrow \tilde{n}(z)$ an extension function of $S \ni y \rightarrow \mathbf{n}(y)$, to an open set of $E_{3}$, containing the crack surface $S$, the second term in the previous kernel on the right-hand side is rewritten as:

$$
\begin{aligned}
\frac{1}{r^{2}} n_{k} n_{i} r_{, j} & =\frac{1}{r}\left(\left.\mathscr{P}_{\operatorname{grad}_{j}}\left(\tilde{n}_{k} \tilde{n}_{i}\right)\right|_{z=y}-\left.\left(\mathscr{P} \operatorname{grad}_{j} \frac{\tilde{n}_{k} \tilde{n}_{i}}{r}\right)\right|_{z=y}+n_{i} n_{j} n_{k} \frac{\mathbf{e}_{r} \mathbf{n}_{y}}{r^{2}}\right. \\
& =\frac{1}{r}\left(n_{k}(y) \sum_{\alpha=1}^{2} \frac{1}{R_{\alpha}}\left(\mathbf{e}_{\alpha}^{y}\right)_{i}\left(\mathbf{e}_{\alpha}^{y}\right)_{j}+n_{i}(y) \sum_{\alpha=1}^{2} \frac{1}{R_{\alpha}}\left(\mathbf{e}_{\alpha}^{y}\right)_{j}\left(\mathbf{e}_{\alpha}^{y}\right)_{k}\right),
\end{aligned}
$$

(using relation (A.5) in the Appendix)

$\left(\left(\mathbf{e}_{\alpha}^{y}\right)_{i}: i\right.$-th component in the fixed basis, of the $\alpha$-th vector of the local basis, $\left.\mathbf{e}_{\alpha}^{y}\right)$.

The third term in the kernel of the right-hand side of (19) can be transformed in quite a similar way. Finally, using the corollary of Stokes' theorem (see relation (A.6) in the Appendix), the left-hand side of (19) becomes:

$$
\begin{aligned}
& \int_{S}\left(\frac{r_{i,} r_{, j}}{r}\right)_{, k} \mathrm{~d}_{y} S=\int_{S}\left\{n_{k}(y) \kappa(y) \frac{r_{, i} r_{, j}}{r}-2 n_{j}(y) \frac{n_{k}(y) n_{i}(y)}{r} \kappa(y)\right. \\
& \quad+2 n_{i}(y) n_{j}(y) n_{k}(y) \frac{\mathbf{e}_{r} \mathbf{n}_{y}}{r^{2}}-3 r_{, i} r_{, j} n_{k}(y) \frac{\mathbf{e}_{r} \mathbf{n}_{y}}{r^{2}}+\frac{1}{r}\left(n_{k}(y) \sum_{\alpha=1}^{2} \frac{1}{R_{\alpha}}\left(\mathbf{e}_{\alpha}^{y}\right)_{i}\left(\mathbf{e}_{\alpha}^{y}\right)_{j}\right. \\
& \left.\left.\quad+n_{i}(y) \sum_{\alpha=1}^{2} \frac{1}{R_{\alpha}}\left(\mathbf{e}_{\alpha}^{y}\right)_{j}\left(\mathbf{e}_{\alpha}^{y}\right)_{k}+n_{j}(y) \sum_{\alpha=1}^{2} \frac{1}{R_{\alpha}}\left(\mathbf{e}_{\alpha}^{y}\right)_{i}\left(\mathbf{e}_{\alpha}^{y}\right)_{k}\right)\right\} \mathrm{d}_{y} S \\
& \quad+\int_{\partial S}\left\{\frac{r_{, i} r_{, j}}{r} v_{k}(y)+\frac{n_{k}(y) n_{i}(y)}{r} v_{j}(y)+\frac{n_{k}(y) n_{j}(y)}{r} v_{i}(y)\right\} \mathrm{d}_{y} l .
\end{aligned}
$$


The proof of Lemma 2 is achieved by noting that:

$$
\begin{aligned}
& \left(\mathbf{a} \mathbf{n}_{y}\right) \mathbf{n}_{y} \wedge\left(\mathbf{n}_{y} \wedge \mathbf{b}\right)+\left(\mathbf{b} \mathbf{n}_{y}\right) \mathbf{n}_{y} \wedge\left(\mathbf{n}_{y} \wedge \mathbf{a}\right)=\mathbf{n}_{y} \wedge\left(\mathbf{n}_{y} \wedge \mathbf{S} \mathbf{n}_{y}\right) \\
& \left(\left(\mathbf{a} \mathbf{n}_{y}\right) \mathbf{n}_{y}-\mathbf{a}\right)(\mathbf{b} \boldsymbol{v})+\left(\left(\mathbf{b} \mathbf{n}_{y}\right) \mathbf{n}_{y}-\mathbf{b}\right)(\mathbf{a} \boldsymbol{v})=\mathbf{n}_{y} \wedge\left(\mathbf{n}_{y} \wedge \mathbf{S} \boldsymbol{v}\right)
\end{aligned}
$$

It should be noted that integral $\mathbf{J}$ is symmetrical and bilinear with respect to $\mathbf{a}$ and $\mathbf{b}$. Moreover, the curvature radii $R_{1}$ and $R_{2}$ appear in both expressions (17) and (18), but they are coupled in the former and uncoupled in the latter which also involves the vectors of the principal basis, $\mathbf{e}_{1}^{y}$ and $\mathbf{e}_{2}^{y}$.

On the strength of Lemmas 1 and 2, singular integrals $v p \mathbf{I}\left(y_{0}\right), v p \mathbf{J}\left(y_{0}\right), y_{0} \in S$, in relation (14) are seen to be transformed into weakly singular ones on $S$. Indeed, as point $x$ in relations (16) tends to point $y_{0}$ of $S$, the integrals containing $1 / r$ in relation (14), (18) (Lemmas 1 and 2) behave like simple-layer potentials and present no particular difficulties. As to the other integrals, their kernels are all proportional to $\mathbf{e}_{r} \mathbf{n}_{y} / r^{2}$ behaving like $r^{2-\alpha}, \alpha>0$. Thus they are merely weakly singular integrals.

So the regularization of relation (7) is achieved and the following proposition holds, which gives the regularized expression of the stress vector $\mathbf{t}\left(y_{0}, \mathbf{n}_{y_{0}}\right)$ at any point $y_{0}$ of the crack:

Proposition: Let $F: \Delta \ni u \rightarrow y \in S$ be a (local or global) parametrization of $S$, defined on a domain $\Delta$ of $\mathbb{R}^{2} . \varphi: S \rightarrow \mathbb{R}^{3}, \phi=\varphi_{0} \mathbf{F}$ be the vector densities defined respectively on $S$ and $\Delta$. If $S$ is of class $C^{2}$ and $\varphi$ of class $C^{1, \beta}(S), 0<\beta \leqslant 1$, then:

$$
\begin{aligned}
& \forall y_{0} \in S, \quad \mathbf{t}\left(y_{0}, \mathbf{n}_{y_{0}}\right)=\frac{E}{16 \pi\left(1-v^{2}\right)} * \int_{\Delta} \frac{1}{r^{2}} \varepsilon_{3 \alpha \beta} \delta\left\{2\left(\mathbf{F}_{, \beta}, \boldsymbol{\phi}_{, \alpha}, \mathbf{e}_{r}\right) \mathbf{n}_{y_{0}}\right. \\
& \quad-(1-2 v)\left[\left(\boldsymbol{\phi}_{, \alpha}, \mathbf{e}_{r}, \mathbf{n}_{y_{0}}\right) \mathbf{F}_{, \beta}+\left(\mathbf{F}_{, \beta} \mathbf{n}_{y_{0}}\right) \phi_{, \alpha} \wedge \mathbf{e}_{r}\right]+3\left(\mathbf{e}_{r} \boldsymbol{\phi}_{, \alpha}\right)\left[\left(\mathbf{F}_{, \beta}, \mathbf{n}_{y_{0}}, \mathbf{e}_{r}\right) \mathbf{e}_{r}\right. \\
& \left.\left.\quad+\left(\mathbf{n}_{y_{0}} \mathbf{e}_{r}\right) \mathbf{e}_{r} \wedge \mathbf{F}_{, \beta}\right]\right\}\left|\mathbf{F}_{, 1}(u) \wedge \mathbf{F}_{, 2}(u)\right| \mathrm{d} u_{1} \mathrm{~d} u_{2}+\varepsilon_{3 \alpha \beta}\left\{2\left(\mathbf{F}_{, \beta}\left(u_{0}\right), \boldsymbol{\phi}_{, \alpha}\left(u_{0}\right), * \mathbf{I}\left(y_{0}\right)\right) \mathbf{n}_{y_{0}}\right. \\
& \quad-(1-2 v)\left(\phi_{, \alpha}\left(u_{0}\right), * \mathbf{I}\left(y_{0}\right), \mathbf{n}_{y_{0}}\right) \mathbf{F}_{, \beta}\left(u_{0}\right)+3\left[* \mathbf{J}\left(\phi_{, \alpha}\left(u_{0}\right), \mathbf{F}_{, \beta}\left(u_{0}\right) \wedge \mathbf{n}_{y_{0}}\right)\left(y_{0}\right)\right. \\
& \left.\left.\quad+* \mathbf{J}\left(\phi_{, \alpha}\left(u_{0}\right), \mathbf{n}_{y_{0}}\right)\left(y_{0}\right) \wedge \mathbf{F}_{, \beta}\left(u_{0}\right)\right]\right\}-\frac{E}{16 \pi\left(1-v^{2}\right)} \int_{\partial S} \frac{1}{r^{2}}\left\{2\left(\tau, \boldsymbol{\varphi}, \mathbf{e}_{r}\right) \mathbf{n}_{y_{0}}\right. \\
& \left.\quad-(1-2 v)\left[\left(\varphi, \mathbf{e}_{r}, \mathbf{n}_{y_{0}}\right) \tau+\left(\tau \mathbf{n}_{y_{0}}\right) \boldsymbol{\varphi} \wedge \mathbf{e}_{r}\right]+3\left(\mathbf{e}_{r} \boldsymbol{\varphi}\right)\left[\left(\tau, \mathbf{n}_{y_{0}}, \mathbf{e}_{r}\right) \mathbf{e}_{r}+\left(\mathbf{n}_{y_{0}} \mathbf{e}_{r}\right) \mathbf{e}_{r} \wedge \tau\right]\right\} \mathrm{d}_{y} l,
\end{aligned}
$$

where $\cdot_{, \alpha}=\partial . / \partial u_{\alpha}, \alpha \in\{1,2\}, r=\left|y-y_{0}\right|, \mathbf{e}_{r}=\left(y-y_{0}\right) / r, \tau$ is the vector tangent to the contour $\partial S$; finally $\mathscr{F}$ being a function of $\boldsymbol{\phi}_{, \alpha}$ and $\mathbf{F}_{, \beta}, \delta \mathscr{F}\left(\phi_{, \alpha}, \mathbf{F}_{, \beta}\right)$ expresses the following difference:

$$
\delta \mathscr{F}\left(\phi_{, \alpha}, \mathbf{F}_{, \beta}\right) \equiv \frac{\mathscr{F}\left(\phi_{, x}(u), \mathbf{F}_{, \beta}(u)\right)}{\left|\mathbf{F}_{, 1}(u) \wedge \mathbf{F}_{, 2}(u)\right|}-\frac{\mathscr{F}\left(\phi_{, \alpha}\left(u_{0}\right), \mathbf{F}_{, \beta}\left(u_{0}\right)\right)}{\left|\mathbf{F}_{, 1}\left(u_{0}\right) \wedge \mathbf{F}_{, 2}\left(u_{0}\right)\right|} .
$$


The expressions of integrals $\mathbf{I}\left(y_{0}\right)$ and $\mathbf{J}(\mathbf{a}, \mathbf{b})\left(y_{0}\right)$ are obtained respectively from relations (17) and (18) by making $x$ tend to $y_{0}$. The asterisk $*$ before these integrals recalls that they are ordinary improper.

Equation (20) constitutes the regularized integral equation for three-dimensional cracks in elastostatics. The line integral along the edge $\partial S$ has the same expression as for the singular integral equation in [9]. It vanishes when the crack is embedded in the infinite medium, whereas in problems of surface cracks or of cracks with a same edge part, it is different from zero. However, on the basis of the expression of the line integral in (19), one can easily show that for the last problems, a set of line integrals the sum of which amounts to zero [14] exists.

\subsection{Case of a flat crack}

If $S$ lies in the plane $P\left(\mathrm{O} ; \mathbf{e}_{1}, \mathbf{e}_{2}\right)$ perpendicular to $\mathbf{e}_{3}$, then for every point $y$ of $S, \mathbf{n}_{y}=\mathbf{e}_{3}$ (which leads us to denote by $\mathbf{n}$ the normal at any point $y$ ), and: $\mathbf{e}_{r} \cdot \mathbf{n}=0, \kappa(y)=0$. Integrals $\mathbf{I}(x), \mathbf{J}(\mathbf{a}, \mathbf{b})(x)$ in relations (16), (17), $x \in E_{3} \backslash S$, reduce to contour integrals:

$$
\begin{aligned}
& \mathbf{I}(x)=-\int_{\partial S} \frac{\boldsymbol{v}}{r} \mathrm{~d}_{y} l, \text { perpendicular to } \mathbf{e}_{3}, \\
& \mathbf{J}(\mathbf{a}, \mathbf{b})(x)=\frac{1}{3} \int_{\partial S}\left[-\left(\mathbf{a e}_{r}\right)\left(\mathbf{b} \mathbf{e}_{r}\right) v+\mathbf{n} \wedge(\mathbf{n} \wedge((\mathbf{b} v) \mathbf{a}+(\mathbf{a} v) \mathbf{b}))\right] \frac{1}{r} \mathrm{~d}_{y} l \\
& r=|y-x| .
\end{aligned}
$$

In the same manner, integrals $* \mathbf{I}\left(y_{0}\right), * \mathbf{J}(\mathbf{a}, \mathbf{b})\left(y_{0}\right), y_{0} \in S$, reduce to contour integrals and are now quite regular.

In the common case when Cartesian coordinates are chosen in plane $P$, i.e. $\left(u_{1}, u_{2}\right)=$ $\left(y_{1}, y_{2}\right)$, one has: $\mathbf{F}_{, \alpha}=\mathbf{e}_{\alpha}, \alpha \in\{1,2\}$. Integral equation (20) now becomes:

$$
\begin{aligned}
& \forall y_{0} \in S, \quad \forall \alpha \in\{1,2\}, t_{\alpha}\left(y_{0}, \mathbf{n}\right)=\frac{E}{16 \pi\left(1-v^{2}\right)} \\
& \times\left\{* \int_{S} \frac{1}{r^{2}}\left\{(1-2 v)\left(\Delta \phi_{\alpha, \beta} r_{, \beta}-\Delta \phi_{\beta, \beta} r_{, \alpha}\right)+3 r_{, \alpha} r_{, \beta} r_{, \gamma} \Delta \phi_{\beta, \gamma}\right\} \mathrm{d} y_{1} \mathrm{~d} y_{2}\right. \\
& \quad+(1-2 v)\left(I_{\beta}\left(y_{0}\right) \phi_{\beta, \alpha}\left(y_{0}\right)-I_{\alpha}\left(y_{0}\right) \phi_{\beta, \beta}\left(y_{0}\right)+3 J_{\alpha}\left(\phi_{, \beta}\left(y_{0}\right), \mathbf{e}_{\beta}\right)\left(y_{0}\right)\right. \\
& -\int_{\partial S} \frac{1}{r^{2}}\left[(1-2 v)\left(\varphi, \mathbf{e}_{r}, \mathbf{e}_{3}\right) \tau_{\alpha}+3\left(\mathbf{e}_{r} \varphi\right)\left(\tau, \mathbf{e}_{3}, \mathbf{e}_{r}\right) r_{, \alpha} \mathrm{d}_{y} l\right\} \\
& \forall y_{0} \in S, \quad t_{3}\left(y_{0}, \mathbf{n}\right)=\frac{E}{16 \pi\left(1-v^{2}\right)} \\
& \quad \times\left\{* \int_{S} \frac{1}{r^{2}} \Delta \phi_{3, \alpha} r_{, \alpha} \mathrm{d} y_{1} \mathrm{~d} y_{2}+I_{\alpha}\left(y_{0}\right) \phi_{3, \alpha}\left(y_{0}\right)-\int_{\partial S} \frac{1}{r^{2}}\left(\tau, \varphi, \mathbf{e}_{r}\right) \mathrm{d}_{y} l\right\}
\end{aligned}
$$


It should be noticed that quantities $\Delta \phi_{i, \alpha}$ appear in (21) instead of $\delta \phi_{i, \alpha}$ in (20):

$$
\Delta \phi_{i, \alpha}=\phi_{i, \alpha}(y)-\phi_{i, \alpha}\left(y_{0}\right)
$$

\section{Conclusion}

The principal value singularity in the expression of the stress vector has been diminished by splitting up the surface integral into two. The former contains the difference between quantities calculated respectively at any point and the singular point, it has been shown under stated hypotheses to be an ordinary improper or weakly singular integral. The strong singularity remaining in the kernel of the latter has been dealt with by use of the specific integrals $I, J$ and this again yields weakly singular integrals.

The regularized expression of the stress vector has been thoroughly expressed in terms of local metrics of a parametrization of the crack surface, which should greatly facilitate its numerical study. However, because of the lengthy expression of the kernel and its complexity, effective numerical investigations can be achieved for the time being only in the case of planar cracks.

\section{Appendix - Operators $\mathscr{P}_{\text {grad }}$ and $\mathscr{D}_{i j}$}

These operators were first introduced in [12] and successfully used in works such as $[3,10,15]$. Consider a real function $\varphi$ defined on surface $S: S \ni y \rightarrow \varphi(y) \in \mathbb{R}$. Partial derivatives of $\varphi$ with respect to Cartesian coordinates, for instance, are meaningless since point $y$ is forced to remain on $S$. Consider in this connection an extension of $\varphi: \tilde{\varphi}: D \ni z \rightarrow \tilde{\varphi}(z) \in \mathbb{R}$, belonging to class $C^{1}(D)$, where $D$ containing $S$ is a domain in the whole space, and $\mathbf{n}$ a unit vector which will become in further applications the normal vector $\mathbf{n}_{y}$ at a point $y$ on surface $S$. The operator "projection of $\operatorname{grad}_{z} \tilde{\varphi}$, parallel to $\mathbf{n}$ " is defined by:

$$
\mathscr{P} \operatorname{grad}(\partial z, \mathbf{n}) \tilde{\varphi}(z)=\operatorname{grad}_{z} \tilde{\varphi}(z)-\frac{\partial_{z} \tilde{\varphi}(z)}{\partial n} \mathbf{n} .
$$

The subscript $z$ should recall that the differentiations must be carried out with respect to variable $z$. From definition (A.1), the operator $\mathscr{D}_{i j}$ is constructed as follows:

$$
\mathscr{D}_{i j}(\partial z, \mathbf{n}) \tilde{\varphi}(z)=(\mathbf{n} \wedge \mathscr{P} \operatorname{grad}(\hat{\partial} z, \mathbf{n}) \tilde{\varphi}(z)) \cdot\left(\mathbf{e}_{i} \wedge \mathbf{e}_{j}\right)=n_{i} \frac{\partial \tilde{\varphi}(z)}{\partial z_{j}}-n_{j} \frac{\partial \tilde{\varphi}(z)}{\partial z_{i}} .
$$

The values obtained from (A.1) and (A.2) at a point $z \in D$ depend obviously on the choice of the extension function $\tilde{\varphi}$. There then arises the question whether their limiting values as $z \rightarrow y \in S$ are actually independent of the choice of $\tilde{\varphi}$. The following theorem in [12] proves to be useful:

Theorem: If $S \in C^{1, \alpha}, 0<\alpha \leqslant 1, \varphi \in C^{1}(S)$, then for any $y \in S$, there exists an application $\tilde{\varphi}$ of the abovementioned type and the limits:

$$
\lim _{z \rightarrow y} \mathscr{P}_{\operatorname{grad}}\left(\partial z, \mathbf{n}_{y}\right) \tilde{\varphi}(z), \quad \lim _{z \rightarrow y} \mathscr{W}_{i j}\left(\partial z, \mathbf{n}_{y}\right) \tilde{\varphi}(z)
$$

do not depend on the choice of the extension $\tilde{\varphi}$. Moreover, these functions of $y$ belong to class $C^{0}(S)$.

The uniqueness of the limiting forms being established, it remains for us to remove the extension $\varphi$ and express the limits in (A.3) in terms of function $\varphi$ itself. With notations introduced for (10), the following proposition holds: 
Proposition: Under the same hypotheses as in the above theorem, we have:

$$
\begin{aligned}
& \lim _{z \rightarrow y} \mathscr{P} \operatorname{grad}\left(\partial z, \mathbf{n}_{y}\right) \tilde{\varphi}(z)=\varepsilon_{3 \alpha \beta} \frac{\partial \phi}{\partial u_{\alpha}^{\prime}}(u) \frac{\partial \mathbf{F}}{\partial u_{\beta}^{\prime}}(u) \wedge \mathbf{n}_{y} \frac{1}{\left|\frac{\partial \mathbf{F}}{\partial u_{1}^{\prime}}(u) \wedge \frac{\partial \mathbf{F}}{\partial u_{2}^{\prime}}(u)\right|}, \\
& \lim _{z \rightarrow y} \mathscr{D}_{i j}\left(\partial z, \mathbf{n}_{y}\right) \tilde{\varphi}(z)=\varepsilon_{3 \alpha \beta}\left[\frac{\partial \phi}{\partial u_{\alpha}^{\prime}}(u) \frac{\partial \mathbf{F}}{\partial u_{\beta}^{\prime}}(u)\right]\left(\mathbf{e}_{i} \wedge \mathbf{e}_{j}\right) \frac{1}{\left|\frac{\partial \mathbf{F}}{\partial u_{1}^{\prime}}(u) \wedge \frac{\partial \mathbf{F}}{\partial u_{2}^{\prime}}(u)\right|} .
\end{aligned}
$$

Application of (A.4) to an extension $\tilde{n}$ of the normal on $S: S \ni y \rightarrow \mathbf{n}(y)$ gives the following proposition:

Proposition: If $S \in C^{2}$, then:

$$
\forall i \in\{1,2,3\}, \lim _{z \rightarrow y} \mathscr{P} \operatorname{grad}\left(\partial z, \mathbf{n}_{y}\right) n_{i}=\sum_{\alpha=1}^{2} \frac{1}{R_{\alpha}}\left(\mathbf{e}_{\alpha}^{y} \cdot \mathbf{e}_{i}\right) \mathbf{e}_{\alpha}^{y},
$$

where $R_{1}, R_{2}$ are the principal curvature radii, and $\left(\mathbf{e}_{1}^{y}, \mathbf{e}_{2}^{y}\right)$ is the principal basis at point $y \in S$.

For the sake of completeness, we give here one corollary of Stokes' theorem [15]:

Proposition: If $S \in C^{2}, \varphi: S \rightarrow \mathbb{R} \in C^{1}(S)$, then $\forall i \in\{1,2,3\}$ :

$$
\int_{S} \lim _{z \rightarrow y} \mathscr{P} \operatorname{grad}_{i}\left(\partial z, \mathbf{n}_{y}\right) \tilde{\varphi}(z) \mathrm{d}_{y} S=\int_{S} n_{i}(y)\left(1 / R_{1}+1 / R_{2}\right) \varphi(y) \mathrm{d}_{y} S+\int_{\partial S} \varphi(y) v_{i}(y) \mathrm{d}_{y} l
$$

where: $v=\tau \wedge \mathbf{n}_{y}, \tau$ is the vector tangent to the crack contour of $S$.

\section{References}

1. T.A. Cruse, International Journal of Solids and Structures 5 (1969) 1259-1274.

2. F.J. Rizzo and D.J. Shippy, International Journal of Numerical Methods in Engineering 11 (1977) 1753-1768.

3. M. Bonnet, "Méthode des équations intégrales régularisées en élastodynamique", thesis, Ecole Nationale de Ponts et Chaussées, November 1986.

4. H.D. Bui, B. Loret and M. Bonnet, Comptes Rendus Academie des Sciences, Paris, t.300, series II, No. 14 (1985) 633-636.

5. B. Loret and M. Bonnet, Congress in Tendances actuelles en calcul de structures, Bastia, November 1985.

6. F.J. Rizzo, D.J. Shippy and M. Rezavat, International Journal of Numerical Methods in Engineering 21 (1985) 115-129.

7. J.B. Leblond, Comptes Rendus Academie des Sciences, Paris, t. 303, series II, No. 17 (1987) 1521-1524.

8. H.D. Bui and M. Bonnet, Regular BIE for three-dimensional cracks in elastodynamics, Symposium IUTAM, San Antonio, April 1987.

9. A. Levan and J. Royer, International Journal of Fracture 31 (1986) 125-142.

10. V. Sladek and J. Sladek, International Journal of Solids and Structures 19 (1983) 425-436.

11. V.D. Kupradze, Dynamical Problems in Elasticity - Progress in Solids Mechanics, vol. III, North Holland (1963).

12. N.M. Gunther, La théorie du Potentiel et ses Applications aux Problèmes Fondamentaux de la Physique Mathématique, Gauthiers-Villars (1934).

13. S.G. Mikhlin, Multi-dimensional Singular Integrals and Integral Equations, Pergamon Press (1965).

14. A. Levan and B. Peseux, International Journal of Numerical Methods in Engineering 26 (1988) 2383-2402.

15. V.D. Kupradze, T.G. Gegilia, M.O. Bashelishvili and T.V. Burchuladze, Three-dimensional Problems of the Mathematical Theory of Elasticity and Thermoelasticity, North Holland (1979). 